\title{
LA MULTI-UNE : COMMENT LES SITES DE PRESSE EN LIGNE S'ADRESSENT AU LECTEUR POTENTIEL
}

\author{
Guillaume Sire ${ }^{1}$
}

A partir d'une étude effectuée dans la rédaction de lexpress.fr, nous analysons la façon dont l'éditeur se saisit des différentes interfaces public/contenu sur le web, selon la marge de manœuvre qui est la sienne, les enjeux économiques et les enjeux de notoriété. Nous montrons quelle est la fonction de chaque type de Une, et comment celles-ci sont assemblées au sein d'un même dispositif stratégique, que nous nommons Multi-Une. Enfin, nous expliquons comment et pourquoi, de la gestion de ce dispositif, sur lequel l'éditeur n'est pas seul à avoir la main, dépend l'inscription des contenus produits par les journalistes à l'avant-scène de l'espace public, considéré comme une échelle de visibilité au sein de laquelle certains propos sont plus publics que d'autres.

Les entreprises de presse traditionnelles ont peu d'interfaces à disposition pour faire la publicité de leurs contenus. Sur Internet, en revanche, elles sont nombreuses. Les agrégateurs, portails, moteurs de recherche, réseaux sociaux, applications, liens hypertextes et newsletters permettent de s'adresser au lecteur potentiel. Le design de ces interfaces n'est pas toujours le fait des éditeurs : il est parfois imposé par la nature même du dispositif. Nous proposons ici de répertorier et

1 Guillaume Sire est Maître de Conférences à l'Université Panthénon-Assas.

Recherches en communication, $\mathrm{n}^{\circ} 39$ (2013). 
de décrire certains exemples significatifs de ces couches de médiation et d'analyser la manière dont l'éditeur peut s'en saisir.

Le pendant empirique de notre recherche est basé sur une étude qualitative effectuée à partir d'entretiens semi-directifs menés entre juin 2011 et mars 2012 auprès des salariés de lexpress. fr : 6 journalistes, rédacteur en chef, éditrice, responsable du référencement, directeur de l'acquisition d'audience, chef d'édition, front page editor, chef de projet et community manager. Le site appartient à Roularta Media France, propriétaire de 21 magazines et réalisant $40 \%$ du chiffre d'affaires du groupe belge Roularta Media Group (725,2 millions d'euros en 2011). Le site lexpress.fr est un site d'informations d'actualités, adossé au magazine hebdomadaire L'Express, dont l'objectif est de traiter l'information en direct. Lancé en 1999, il n'avait en 2005 qu'un rédacteur en chef, chapeautant un journaliste et deux stagiaires (Smyrnaios, 2005, p.387). En 2012, il compte une vingtaine de journalistes. Son modèle d'affaires est essentiellement basé sur la publicité display, facturée au coût par affichage. Son audience se situe entre 5 et 5,5 millions de visiteurs uniques par mois, ce qui en fait le huitième site d'information politique et générale selon l'OJD (décembre 2012). La structure d'audience, quant à elle, nous a été décrite comme étant la suivante : $25 \%$ provenant de Google Actualités, $25 \%$ provenant de Google Search ${ }^{1}, 20 \%$ d'accès directs, $15 \%$ de sites influents, $7 \%$ de partenariats avec Yahoo et Orange, environ 2-3\% Facebook et moins de $1 \%$ Twitter.

Nous avons choisi lexpress.fr car ses dirigeants ont œuvré à la rationalisation de la gestion des interfaces public/contenu, en embauchant des personnels à ce seul effet, à l'instar de leurs principaux concurrents (lefigaro.fr, lemonde.fr, leparisien.fr, lenouvelobs.com, lepoint.fr et 20minutes. fr) dont les modèles d'affaires sont eux aussi basés sur la publicité facturée au coût par affichage. Notre étude ne permet donc pas de tirer des conclusions quant aux sites qui n'ont pas rationalisé la gestion de leurs interfaces, soit parce qu'ils n'en avaient pas les moyens, soit parce que leurs modèles d'affaires ne s'appuient pas sur la facturation au coût par affichage (et donc sur la maximisation du nombre de visiteurs uniques et de pages vues). Quant aux sites

1 Dans ces chiffres, les internautes qui proviennent de Google en ayant tapé la requête « l'express » ou « l'express.fr » ne sont pas compatibilités. Ils sont comptabilisés dans les accès directs. 
comparables à lexpress.fr, notre étude ne permet que d'émettre des hypothèses à leur sujet, et nécessitera d'être poursuivie.

\section{La vitrine des contenus journalistiques sur le web}

Sur le web, les voies d'accès par lesquelles des lecteurs arrivent sur le site d'un éditeur sont nombreuses. On estime à la moitié la proportion de visiteurs qui arrivent sur un site de presse indirectement (Foster, 2012). Il semblerait ainsi que la vitrine de la presse en ligne soit un prisme dont chaque facette a des caractéristiques différentes quant à la dilatation et la diffraction de la lumière. Dans le cadre de notre étude, nous avons choisi de répertorier les principales interfaces selon une typologie comprenant trois sous-groupes distincts : les Unes maîtrisées, semi-maîtrisées et non-maîtrisées. Cette typologie a une vocation heuristique plutôt qu'exhaustive : elle nous sert à comprendre comment le dispositif stratégique que nous nommerons Multi-Une peut faire l'objet d'une rationalisation, et ce même si les éditeurs ne peuvent exercer à son endroit qu'un contrôle partiel.

\section{Les Unes maîtrisées}

La page d'accueil d'un site web et les applications mobile/tablette sont des Unes maîtrisées. Certes, la technique, étant donné ce qu'elle permet, joue un rôle dans leur définition, mais il n'en demeure pas moins que les dirigeants et les employés de l'entreprise de presse décident de leur apparence et de leur contenu. Ils choisissent dans quel ordre apparaissent les articles, sous quel format, avec ou sans image, les rubriques, les polices d'écriture, le CMS de publication, le système de tagging, les modalités d'accès aux archives, l'emplacement des bannières publicitaires. Ces Unes mêlent les choix faits par la rédaction (sous l'autorité du rédacteur en chef), les choix des équipes marketing (sous l'autorité de l'éditrice) et les choix des équipes techniques (sous l'autorité du rédacteur en chef et de l'éditrice).

Sur le site web et les applications, nous pouvons tester différentes solutions, arbitrer comme nous le voulons, proposer aux annonceurs des opérations spéciales. C'est finalement assez semblable à ce qu'était la Une d'un journal papier. On fait ce qu'on veut, du moment que ça fonctionne. Et si demain on 
voit que ce n'est plus efficace, on peut changer la présentation, l'ordre des rubriques, etc.

(éditrice)

A cela, il faut ajouter les articles eux-mêmes, en général considérés comme des Unes à part entière. Chacun d'eux est accompagné d'images et de liens visant à envoyer le lecteur sur d'autres pages du site. Là encore, l'environnement est maîtrisé : c'est l'éditeur qui choisit, selon ce qu'il peut mettre en œuvre techniquement, quels articles remonteront, combien, comment et où.

A une époque, on faisait des sommaires sur chaque page article où on mettait les $5 / 6$ derniers papiers sur le même sujet. Maintenant, je préfère en mettre un ou deux bien choisis, bien anglés, plutôt que la première dépêche bâtonnée sur le sujet. (front page editor)

Toujours dans ces Unes maîtrisées, on trouve les alertes et les newsletters, envoyées par la rédaction aux abonnés pour les prévenir qu'un sujet brûlant a été traité ou leur signifier que de nouveaux articles ont été mis en ligne. Ce sont les équipes techniques, la rédaction, et les équipes marketing et commerciale qui décident de l'apparence de ces alertes et newsletters, choisissent la hiérarchie des informations et les modalités de l'affichage publicitaire. La rédaction se charge en particulier de ce qui est éditorial et de la fréquence d'envoi.

C'est la rédaction qui décide d'envoyer une alerte sur un sujet. Tu as des journaux qui vont envoyer dix alertes dans la journée, d'autres une, d'autres jamais. Il faut trouver un équilibre à propos de quoi on envoie des alertes, et comment : une phrase façon breaking news, ou bien un truc plus long, quand on a une grosse info ou un papier à valeur ajoutée. On en discute en permanence, car on ne veut pas avoir l'air de « spammer» les abonnés.

(front page editor)

Les Unes maîtrisées sont entièrement intégrées à la ligne éditoriale. Elles s'adressent au lecteur qui se trouve sur le site, ou bien à celui qui a émis explicitement son intérêt en s'abonnant à la newsletter et/ou aux 
alertes. En cela, elles sont comparables aux Unes de la presse imprimée et aux campagnes de publicité par courrier.

\section{Les Unes semi-maîtrisées}

Certaines vitrines de la presse en ligne se situent sur d'autres sites que le site appartenant à l'éditeur et, dès lors qu'il s'en saisit, celuici, même s'il peut choisir ce qu'il met en ligne, doit composer avec des contraintes techniques et graphiques qui lui sont imposées, ainsi qu'avec certaines règles dont le respect conditionne sa présence dans ces espaces. C'est le cas des réseaux sociaux Facebook et Twitter. Sur ces sites, l'éditeur peut créer un compte, mettre ses propres illustrations, poster des liens et du texte, mais il n'aura qu'un contrôle partiel sur l'apparence de sa page et des contenus qu'il y positionne. En outre, il change la manière dont il s'adresse aux lecteurs :

On ne s'adresse pas à nos communautés Facebook et Twitter comme on s'adresse aux lecteurs sur notre site. On leur parle avec un ton plus libre, comme à des copains ou des collègues, on s'autorise les jeux de mots, un ton «plus jeune ». On leur répond. C'est surtout vrai sur Twitter. Il faut écrire comme ça sur Twitter et Facebook, sinon ça ne marche pas.

(community manager)

La pluparts des sites de presse ont leur page Facebook. On y trouve en général des photos, titres et accroches d'articles publiés, dans l'ordre antéchronologique. Lexpress.fr en a rationnalisé l'usage selon onze créneaux de publication. Le premier de la journée est un récapitulatif des actualités survenues durant la nuit. Pour chaque autre créneau, un arbitrage est effectué par le community manager qui choisit parmi les derniers articles publiés lequel, ou lesquels, il mettra en avant. Ainsi, une dissociation est faite en termes d'horaires de publication entre le site lexpress.fr et la page Facebook :

Il y a des moments dans la journée, par exemple si on a une info très importante qui arrive, où il vaut mieux qu'on retarde la publication sur Facebook. On ne le fait pas sur le site, mais 
uniquement sur Facebook, parce que sur le créneau de 15h, on sait que l'info sera davantage visible.

(community manager)

A part le premier créneau de la journée, un créneau n'est pas alloué spécifiquement à un type de sujet. Cependant, certains critères sont pris en compte : par exemple, s'il n'y a pas d'actualité brûlante, les sujets « télé » sont privilégiés à $20 \mathrm{~h}$.

On a remarqué que sur Facebook, si on postait un message qui concerne une émission télé, un film qui va passer par exemple, les gens ont tendance à commenter l'émission pendant qu'ils la regardent. Ça donne des choses intéressantes, parce qu'ils sont directement concernés par ce qu'on met et ils ont envie de participer. C'est pour ça qu'on privilégie les sujets télé à $20 \mathrm{~h}$. (community manager)

La page Facebook apporte une audience assez faible au site lexpress.fr. Mais l'éditrice et le rédacteur en chef nous ont expliqué qu'il était « inconcevable » de ne pas y figurer. Il semble ainsi que la présence sur le réseau social soit d'abord liée à l'image du titre, plutôt qu'à des raisons économiques. Cela tient à une certaine « obligation d'évoluer » (Zouari, 2007) et non au calcul rationnel d'un point de vue économique. De la même manière, les sites web, dans les années 90 , avaient été créés par les titres de presse souvent dans le but de montrer qu'ils étaient présents sur ce nouvel espace (Antoine, 1998).

La plupart des membres du groupe Facebook ne consultent jamais, ou très peu, les articles sur le site lexpress.fr. Plutôt que de chercher à les appâter, le community manager nous a expliqué qu'il essayait de leur donner le plus d'informations possible, pour qu'ils soient tout de même informés sans s'être rendus sur lexpress.fr. Facebook n'est donc pas un moyen de générer des revenus, mais un moyen de faire la publicité de la marque en établissant une relation privilégiée avec certains usagers du réseau, quitte à ce que les informations mises en ligne par ce biais se substituent aux informations du site.

La majorité des lecteurs de la page Facebook ne vont jamais sur la page de lexpress.fr. Par exemple, sur 6000 personnes qui ont vu un lien, entre 300 et 500 y cliqueront. C'est pour ça qu'il faut qu'on bosse nos accroches, parce qu'il faut donner l'info 
essentielle, car de toute façon ils n'iront pas la lire. En termes de trafic, donc, ce n'est pas important, mais en termes d'image, il faut qu'on soit sur Facebook. Ça nous permet d'établir une relation avec un autre type de lecteurs. [...] Mais ce n'est pas un vecteur d'audience véritablement, pas pour le moment en tout cas.

(community manager)

La Une semi-maîtrisée sert à renforcer l'image du titre et permet de s'adresser à des lecteurs qui de toute façon n'auraient pas été atteints via les Unes maîtrisées. Elle est un moyen pour les journalistes de remplir ce qu'ils estiment être leur mission d'information. Par ailleurs, le ton, sur Facebook, est plus souple que sur lexpress.fr :

Sur Facebook, on est plus libre, si je veux mettre des commentaires, présenter l'actualité d'une certaine façon, interpeler un membre, je le fais avec un ton correspondant à ceux à qui je m'adresse, qui n'est pas toujours le même ton que celui de la ligne éditoriale du site.

(community manager)

Le community manager nous a également expliqué qu'il n'était pas soumis au circuit de validation auquel sont soumis les journalistes, bien qu'il soit lui-même journaliste. Il peut, par exemple, poster directement les liens qu'il souhaite. Ainsi, alors que les Unes maîtrisées sont entièrement intégrées à la ligne éditoriale, les Unes semi-maîtrisées peuvent faire l'objet d'un traitement différent, en termes notamment de ton employé, de circuit de validation et de créneaux de publication. Cela est principalement dû, selon les employés de lexpress.fr, au lectorat à qui s'adresse la page Facebook, qui diffère du lectorat du site.

Sur Twitter, lexpress.fr possède un compte alimenté par le community manager. Chaque journaliste possède également son propre compte, qu'il alimente comme il le souhaite. La publication n'y est pas rationnalisée par tranches horaires comme sur Facebook. Cependant, nous retrouvons certaines caractéristiques propres à ce que nous avons nommé la Une semi-maîtrisée : les auteurs sont limités à 140 caractères, ils n'ont pas la main sur l'interface graphique et ils s'adressent à un public en particulier (Rieder, Smyrnaios, 2011), réunis dans ce que l'on peut nommer une « communauté de pratique » (Weiss, Domingo, 2010). L'enjeu en termes économiques est faible, étant donné qu'à peu près 
$1 \%$ de l'audience du site arrive par Twitter, alors que chaque journaliste interrogé nous a dit twitter au moins cinq fois par jour. La présence sur Twitter est donc encore une question d'image. Et Twitter est également utilisé par les journalistes comme un outil de veille informationnelle.

Il existe d'autres Unes semi-maitrisées, comme le dispositif mis en place par Google sur son agrégateur Google Actualités : « Le choix des éditeurs ». Ce module permet à chacun des titres sélectionnés de mettre en avant cinq articles de son choix. Là encore, nous retrouvons les mêmes caractéristiques : un enjeu d'audience assez faible (inférieur à $0,5 \%$ ) et un enjeu d'image mis en avant :

Pour qui passerions-nous auprès de la concurrence si nous n'y étions pas?

(journaliste)

\section{Les Unes non-maîtrisées manuelles}

Certaines voies d'accès échappent presque entièrement à l'éditeur. Parmi elles, il convient d'en dissocier au moins deux sortes : la première étant le fait d'un traitement manuel effectué par des personnes qui ne sont pas des employés de l'entreprise de presse, la seconde étant le fait d'un traitement automatique.

Dans la première catégorie, une des voies d'accès les plus importantes est Yahoo. Le portail noue des partenariats avec les éditeurs, qui lui donnent l'autorisation de reprendre leurs contenus, soit contre une somme d'argent, soit sur une base d'échange de visibilité contre contenu (Attias, 2007). Les contenus sont mis en forme par Yahoo, qui en modifie le format selon sa charte graphique. Les employés du portail choisissent quels contenus ils reprennent (Smyrnaios, Boure, 2006). Un lien est mis en bas de chaque article vers la source originale, pour les lecteurs qui voudraient lire la suite et trouver l'information dans son contexte original.

Les éditeurs dont les contenus sont repris sur Yahoo reçoivent une très forte audience par ce biais. Ils ont donc intérêt à nouer ce partenariat, et ce même si Yahoo ne les paye pas et si une partie du lectorat se contente des contenus du portail, qui se substitue alors au site. En général, le texte des articles n'est pas changé, simplement son format : le contenu repris est en adéquation avec la ligne éditoriale de l'éditeur. Il peut arriver que les employés de Yahoo modifient une partie du titre ou de l'accroche pour l'adapter à leur lectorat, mais ces cas, 
selon le rédacteur en chef de lexpress.fr, sont très rares. Même s'ils n'ont pas la main sur les articles repris par Yahoo, la rédaction entretient des relations avec les employés du portail, par téléphone, mail et Twitter, de manière à les prévenir quand un article est susceptible de les intéresser, dans l'espoir que celui-ci soit ensuite repris. Ainsi, ce n'est pas parce que la Une est non maîtrisée qu'elle ne donne lieu à aucune action : au contraire, étant donné l'enjeu d'audience, l'éditeur fait ce qu'il peut pour l'optimiser.On trouve également dans les Unes non maitrisées manuelles l'écosystème de la recommandation. Grâce au lien hypertexte, les éditeurs professionnels et amateurs peuvent pointer vers le contenu d'un éditeur et lui apporter ainsi une audience qui peut être minime ou significative. Lexpress. fr reçoit par ce biais 7 à $8 \%$ de son audience. Cette voie échappe entièrement au contrôle de l'éditeur, dans la mesure où il ne choisit ni l'ancre ni son contexte. C'est le jeu du web : n'importe qui peut faire un lien vers n'importe qui d'autre, sans que ce dernier ne puisse l'en empêcher (Barabasi, 2003).

Enfin, on trouve les dispositifs de recommandation tels que le like de Facebook. Cette fois, c'est sur la page Facebook d'un internaute, ou d'un autre éditeur, qu'apparaîtra un lien vers un article de lexpress.fr. Là encore, l'enjeu d'audience est important : le community manager nous a dit que lexpress.fr pouvait parfois recevoir jusqu'à $10 \%$ de son trafic via ce biais. Là encore, l'éditeur ne maîtrise pas les conditions dans lesquelles le lien sera posté. Il ne peut qu'espérer faire l'objet de recommandations nombreuses.

\section{Les Unes non-maîtrisées automatiques}

Dans les Unes non-maîtrisées automatiques, on trouve principalement les moteurs de recherche et les agrégateurs. L'éditeur n'a en effet pas la main, ou très peu, sur la reprise de ses contenus et leur apparence. L'enjeu d'audience, ici, est considérable. Notamment, Google Search (moteur généraliste) et Google Actualités (moteur spécialisé) apportent en moyenne $50 \%$ de son trafic à lexpress.fr. Le référencement joue donc un rôle économique extrêmement important, quasiment aussi important que la notoriété du titre (Sonnac, 2009). Cette situation de dépendance vis-à-vis de Google a créé de nombreuses frictions entre les éditeurs de presse et la firme de Mountain-View, ceux-là reprochant à celle-ci de reprendre sur ses pages un contenu qui ne lui appartient pas. L'affaire a plusieurs fois été portée devant les tribunaux (Manara, 2011). Comme pour le cas de Facebook et de Yahoo, les contenus repris sur 
ces Unes peuvent se substituer aux contenus du site et ne générer aucun profit : selon une étude réalisée en 2010 par la compagnie Outsell, 44\% des utilisateurs américains de Google Actualités lisent les titres et les accroches sans cliquer sur les liens (Wauters, 2010).

Même s'ils ne maîtrisent pas cette voie d'accès, les éditeurs peuvent décider de mettre en œuvre des solutions pour tâcher d'en optimiser l'apport, grâce aux techniques dites de Search Engine Optimization (SEO). Lexpress.fr a engagé en 2009 un spécialiste du référencement, chargé de mettre en œuvre des solutions techniques et éditoriales afin d'optimiser l'audience provenant de Google. L'aspect éditorial de son travail consiste principalement à former les journalistes : leur enseigner quels mots privilégier dans le titre et l'accroche, quel usage faire des liens, quelle temporalité de publication adopter, comment écrire ses intertitres.

Le référenceur a un poste clef et surtout il doit passer les bons messages aux bonnes personnes : il ne peut pas y arriver tout seul, parce que c'est autant de la technique que du rédactionnel : la façon dont on écrit pour Google. (éditrice)

Le responsable SEO a pris plusieurs mesures techniques, parmi lesquelles le dédoublement du titre : le CMS de publication permet d'écrire un titre pour Google qui sera repris sur les moteurs, et un titre pour le lecteur, qui apparaîtra sur la page article.

Le titre SEO est une bonne trouvaille parce que ça permet de concilier travail éditorial et travail de référencement. [...] On peut s'amuser et faire quand même potentiellement des titres drôles en mettant un titre SEO très informatif.

(journaliste)

J'utilise le titre SEO quand ce sont des papiers très travaillés, pour lesquels j'ai enquêté plusieurs jours. D'une part donc, on a envie de signer le titre et puis aussi c'est un signal lancé à l'internaute : l'internaute qui va voir un titre avec un jeu de mots, il va se dire : ce n'est pas une dépêche, ça va plus loin, c'est autre chose.

(journaliste) 
Nous constatons ici comment le journaliste, concerné par la publicisation de son contenu, n'est pas gêné par ce que pourrait lui imposer une écriture SEO. L'optimisation de la Une non-maîtrisée ne prend pas le pas sur l'élaboration de la Une maîtrisée. Cependant, certains journalistes nous ont dit être méfiants vis-à-vis des consignes qui leur sont données. Ils ne sont pas prêts à faire «n'importe quoi », et il arrive par conséquent qu'il y ait certaines « frictions » avec le référenceur :

Je ne veux pas travailler uniquement pour Google et je veux que ma Home Page ressemble quand même à quelque chose. Avec [le référenceur], de temps en temps, il y a des petites frictions, parce que lui il fait son job, il essaye des choses, il essaye de comprendre, mais nous aussi on fait notre job.

(front page editor)

Parfois, il vient nous voir et il nous demande de faire des opérations, des trucs, qui n'ont pas de sens éditorial, donc on ne le fait pas forcément.

(rédacteur en chef adjoint)

Ça peut être chaud pour les journalistes, parfois. Mais j'essaye quand même de leur montrer que ça vaut le coût.

(référenceur)

L'éditrice nous a expliqué que certains projets, qui pourraient être éventuellement intéressants sur le plan éditorial, n'étaient pas réalisés parce qu'ils n'avaient « aucun potentiel SEO ». Quant aux journalistes, certains redoutent les effets d'une telle stratégie :

Il y a beaucoup trucs qu'on n'aurait jamais faits s'il n'y avait pas Google. Pour le choix des sujets notamment : tu as la pression de Google, et tu as la pression des concurrents qui sont eux-mêmes soumis à Google. C'est-à-dire qu'un concurrent va se dire : « ça marche bien sur Google » et il va le faire. Et un autre va se dire «mon voisin l'a fait, ça marche, je vais le faire aussi » et ça se répandra un peu comme ça. Sur le choix des sujets, c'est donc clair qu'il y a une influence de Google. (journaliste)

La rationalisation pourrait donc faire peser une menace sur ce que les journalistes estiment être la qualité du contenu qu'ils produisent 
et, de manière plus générale, sur ce qu'ils estiment être leur mission d'information.

\section{Conclusion : la Multi-Une et ses enjeux}

Nous avons expliqué comment ce qui était une vitrine dans le cas de la presse imprimée est devenue un prisme sur le web, dont les facettes sont différentes les unes des autres. Les interactions lecteur/contenu peuvent avoir lieu dans des conditions très hétérogènes, notamment selon que l'éditeur en choisit directement, ou non, les composantes. Nous avons identifié au moins trois types de facettes :

- Les Unes maîtrisées ressemblent à ce qui existait déjà dans le cas de la presse imprimée, à la différence près qu'elles sont plus nombreuses et peuvent faire l'objet de temporalités de publication différentes. Elles s'adressent à un lectorat fidèle.

- Les Unes semi-maîtrisées, associées à des « services d'usage massif » (Rieder, Smyrnaios, 2011), apportent peu d'audience au titre, mais elles sont là pour des questions d'image, et pour atteindre certaines communautés (Twitter) ou certains lecteurs qui n'auraient pas été atteints sinon (Facebook). Leur ligne éditoriale peut différer - ton employé, hiérarchie des articles, temporalité de publication - de celle du titre auquel elles sont affiliées. Elles peuvent également faire concurrence au contenu lui-même, et cela sans surprise, car elles sont volontairement conçues comme des substituts.

- Les Unes non-maîtrisées apportent une audience considérable, au moins aussi importante, si ce n'est plus, que les Unes maîtrisées. Comme dans les Unes semi-maîtrisées, elles peuvent se substituer aux Unes maîtrisées et au contenu du site web. Les éditeurs mettent en place des partenariats ou des politiques SEO pour optimiser cette vitrine, sur laquelle, contrairement aux réseaux sociaux, aux newsletters et aux alertes, ils n'ont pas la main. Leurs actions vis-à-vis de ces Unes sont faites dans l'espoir de recevoir du trafic. Enfin, les lecteurs qui arrivent par ce biais ne constituent pas, ou pas encore, un lectorat fidèle. 


\begin{tabular}{|c|c|c|c|}
\hline 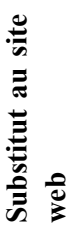 & 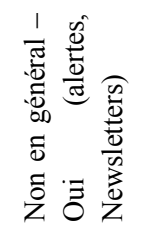 & $\ddot{z}$ & $\bar{z}$ \\
\hline 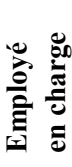 & 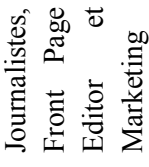 & 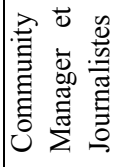 & 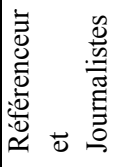 \\
\hline & 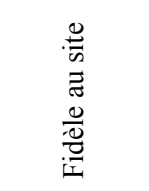 & 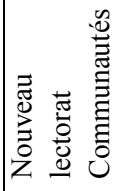 & 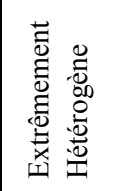 \\
\hline 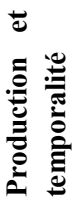 & 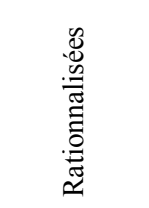 & 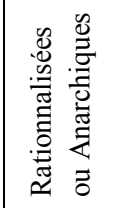 & 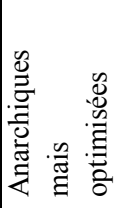 \\
\hline 莺 & $\frac{\pi}{\overrightarrow{0}}$ & $\begin{array}{l}\stackrel{0}{0} \\
\stackrel{0}{0} \\
\stackrel{\circ}{n}\end{array}$ & $\underset{\Xi}{\Xi}$ \\
\hline : & 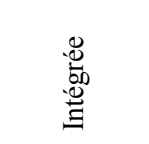 & 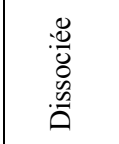 & 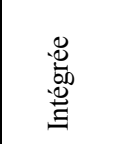 \\
\hline 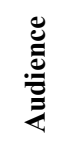 & $\begin{array}{l}\stackrel{0}{ } \\
\text { 仓े } \\
\text { ঠे }\end{array}$ & 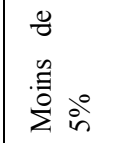 & $\begin{array}{l}\text { oे } \\
\text { iे } \\
\text { iे }\end{array}$ \\
\hline \multirow[t]{2}{*}{$\stackrel{\vec{\Xi}}{\vec{\Xi}}$} & 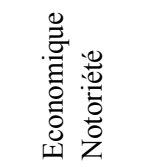 & $\begin{array}{l}\frac{0}{0} \\
: \frac{0}{0} \\
0 \\
0\end{array}$ & 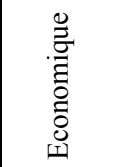 \\
\hline & 串 & | & 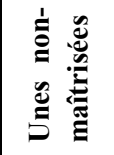 \\
\hline
\end{tabular}


Il existe des correspondances entre les Unes. Google prend par exemple en compte dans son référencement le nombre de like sur Facebook et le nombre de fois qu'un article a été twitté. Google prend également en considération les liens hypertextes entre les documents. Ainsi, grâce à des actions effectuées vis-à-vis des Unes maîtrisées et semi-maîtrisées, l'éditeur peut diminuer l'incertitude entourant l'exposition de la Une non-maîtrisée. En outre, le but pour les éditeurs est qu'un internaute arrivé via une Une non-maîtrisée sur une page article lise ensuite plusieurs articles, puis qu'il revienne, s'abonne et télécharge l'application : il sera ainsi davantage exposé aux Unes maîtrisées. La lumière qui passe par une des facettes du prisme peut ainsi augmenter la taille, l'exposition et l'attrait d'une autre facette. Celles-ci sont interdépendantes, réunies autour du Tout que forme le contenu produit par les journalistes et les publicités des partenaires. C'est ce prisme que nous appelons Multi-Une.

Grâce à la bonne gestion de la Multi-Une, un éditeur peut inscrire ses documents en haut de ce que Cardon (2010) nomme "l'échelle de visibilité collective » : espace conçu comme un continuum au sein duquel certains propos sont plus publics que d'autres. Et nous avons vu que la rationalisation de la gestion de ces interfaces demande des efforts importants, notamment en termes de coûts : un employé (le front page editor) de lexpress.fr ne s'occupe que des Unes maîtrisées, un employé (le community manager) des Unes semi-maîtrisées, et un employé (le référenceur) des Unes non-maîtrisées. Les journalistes, le rédacteur en chef, les chefs d'éditions et l'éditeur contribuent également à assurer la bonne gestion de la Multi-Une. Dès lors, il semblerait qu'une telle logistique assure à ceux qui en ont les moyens de demeurer dans à l'avant-scène de l'espace public, loin devant les amateurs.

Dans un tel contexte, il sera intéressant de questionner les effets de la gestion de la Multi-Une sur la qualité du contenu produit et sur la mission d'information du journaliste. En effet, nous avons vu que la rationalisation et l'optimisation, notamment dans le cas des Unes nonmaîtrisées, peuvent influencer le contenu lui-même, la ligne éditoriale, la hiérarchisation de l'information, les mots choisis. Un arbitrage doit donc être fait par l'éditeur s'il souhaite maîtriserau mieux les canaux de diffusion tout en conservant la maîtrise de sa ligne éditoriale.

Par ailleurs, la question de pose de savoir si la typologie et les éléments dégagés peuvent être généralisés à l'ensemble des éditeurs. C'est la principale limite de notre travail et, plus généralement, la carence de toute approche monographique. C'est pourquoi il nous 
faudra poursuivre notre étude auprès d'autres rédactions, dans le but d'épaissir, et peut-être de modifier, notre typologie.

\section{Références}

Antoine, F. (1998). Production journalistique et journaux en ligne. Les Cahiers $d u$ Journalisme, 5.

Attias, D. (2007). L'impact d'Internet sur l'économie de la presse : quel chemin vers la profitabilité ? (Thèse de Sciences Economiques, Université de Paris-X Nanterre). Disponible à : http://danielleattias.typepad.com/medias/files/These_Danielle_ Attias.pdf.

Barabasi, A.-L. (2003). Linked. How Everything is Connected to Everything Else and What it Means for Business, Science and Everyday Life. Cambridge : A Plume Book.

Cardon, D. (2010). La démocratie Internet. Promesses et limites. Paris : Le Seuil.

Manara, C. (2011). Le droit d'auteur contre l'accès à l'information mondiale ? Le cas des moteurs de recherche. Revue internationale de droit économique, 25, pp.143164.

Foster R. (2012), «News Plurality in a Digital World», Reuters Institute Digital News Report, 2012, disponible à : https://reutersinstitute.politics.ox.ac.uk/fileadmin/ documents/Publications/Working_Papers/News_Plurality_in_a_Digital_World.pdf Rieder, B., \& Smyrnaios, N. (2011). Les journalistes français sur Twitter: une étude exploratoire. Communication lors de la Journée d'étude Sources et flux des news/ nouvelles, Université Sorbonne Nouvelle. Disponible à : http://epresence.univparis3.fr/1/watch/126.aspx

Smyrnaios, N. (2005). L'industrie de l'éphémère. Emergence et consolidation de modèles diversifies de production et de diffusion de l'information en ligne. (Thèse en Sciences de l'information et de la communication, Université Grenoble 3).

Smyrnaios, N., \& Boure, R. (2006). L'infomédiation de l'information en ligne, le cas des filiales françaises de Google et Yahoo. Document numérique et société, Paris : ADBS Éditions, pp.43-55.

Sonnac, N. (2009). L'Economie de la presse : vers un nouveau modèle d'affaires. Les cahiers du journalisme, 20.

Wauters, R. (2010). Report: 44\% Of Google News Visitors Scan Headlines, Don't Click Through. TechCrunch, disponible à : http://techcrunch.com/2010/01/19/outsellgooglenews/

Weiss, A., \& Domingo, D. (2010). Innovation processes in online newsrooms as actornetworks and communities of practice. New Media \& Society, 12(7), 1156-1171.

Zouari, K. (2007). La presse en ligne : vers un nouveau média ? Les enjeux de l'information et de la communication, disponible à : http://w3.u-grenoble3.fr/les_ enjeux/2007/Zouari/index.php 